\title{
Model Diatom Population by Branching Stochastic Processes
}

\author{
Antoanela Terzieva*, Georgi Terziev \\ Department of Probability, Operations Research and Statistics, Sofia University “St. Kliment Ohridski”, Sofia, Bulgaria
}

Email address:

antoanela.t@abv.bg (A. Terzieva),gterziev@gmx.net (G. Terziev)

${ }^{*}$ Corresponding author

\section{To cite this article:}

Antoanela Terzieva, Georgi Terziev. Model Diatom Population by Branching Stochastic Processes. Applied and Computational Mathematics. Vol. 8, No. 1, 2019, pp. 3-8. doi: 10.11648/j.acm.20190801.12

Received: December 14, 2018; Accepted: January 11, 2019; Published: February 21, 2019

\begin{abstract}
The phytoplankton is one of the most ancient inhabitants of our planet. It consists of mostly unicellular aquatic species, both fresh water and marine. The purpose of this work is to model the dynamics of a diatoms population because it is a predominant phytoplankton kind and plays a key role at the base of the food chains, climate regulation and ecology. The formulated mathematical model would give a better idea about the expected population size in the near and further future. As a modelling tool we propose the branching stochastic process of Bellman-Harris (BPBH) Z (t). In general, the generating function (g.f.) $\mathrm{F}(\mathrm{t})$ for non Markov multidimensional BPBH is difficult for explicit expression. Impossibility for simultaneous birth and death of the BPBH-particle together with producing offspring would correspond to the biological side. Only after completion of the whole cycle the cell is capable of dividing and every particle is of zero age at birth, which corresponds to the condition of right continuity at the zero point of the distribution function (d.f.) $G(t)$. It makes the multidimensional g.f. $F(t)$ more suitable for research and analytical expression, allowing the use of basic theorems. The matrix $U(t)$ of means meets the requirements and satisfies the basic matrix equation for a multidimensional non Markov branching processes. The matrix equation, corresponding to the system of sixteen integral equations is determined. The moments of $Z(t)$ are expressed. The most characteristic feature of the diatoms is their cell wall - the cause of mitosis to result in one of the two daughters decreasing in size. This again directs the authors to determine the particle's type by its initial size and model by suggesting a decrease in the offspring size. The diatom's cell stops dividing when their size drops below the minimum. Accumulating sufficient critical mass, cells that have ceased to divide begin to merge with each other, generating a new cell. In contrast to the determined models the stochastic processes assess the probable future development. A certain fact is that the diatoms number is influenced by many factors of random nature in the environment.
\end{abstract}

Keywords: Cell Division, Diatoms, Phytoplankton, Bellman-Harris, Chlorophyll, Non Markov Multidimensional Stochastic Process

\section{Introduction}

About half of all Earth's photosynthesis is due to phytoplankton. It produces over 80 percent of oxygen. On the Bulgarian Black See coast there are about 600 species phytoplankton. Phytoplankton consists of about 16000 microscopic aquatic species, both fresh water and marine. Diatoms are the most successful phytoplankton group in the modern ocean and have risen to dominance relatively quickly over the last 100 million years $[1,12]$. The phytoplankton plays a key role at the base of the food chains, climate regulation and ecology [3]. The branching stochastic processes assess the probable future development $[2-4,7]$. The phytoplankton, in particular the diatoms are photosynthetic and therefore contain chlorophyll-a (chl-a) [5, 12]. Its quantity increases and divides together with the whole cell. Data about chl-a can be easily acquired via satellite. The chlorophyll-a concentration can be a measure of the phytoplankton concentration. The proposed model allows to interpret as a particle not the whole cell, but only the "unit" of 
chl-a contained in it $[6,8]$. In the considered $\mathrm{BPBH}$ it is impossible simultaneous birth, death and offspring-production of the particle. Every particle is of zero age at birth. This allows the use of some basic theorems for multidimensional continuous non-Markov branching processes. Every particle has a life span $\tau$ and produces offspring just before dying. These two conditions completely coincide with the diatom's cell-division, which is one of the reasons why the authors model through BPBH. The mathematicians have long ago discovered that in discrete time the Fibonacci series describes successfully the diatoms' number $0,1,1,2,3,5,8,13 \ldots .[1$, 12] The particle's type is defined by its initial size $[6,8]$. Phytoplankton feeds even the blue whale, which is considered to be the greatest animal ever lived on our planet. The next picture presents different kinds of diatoms.

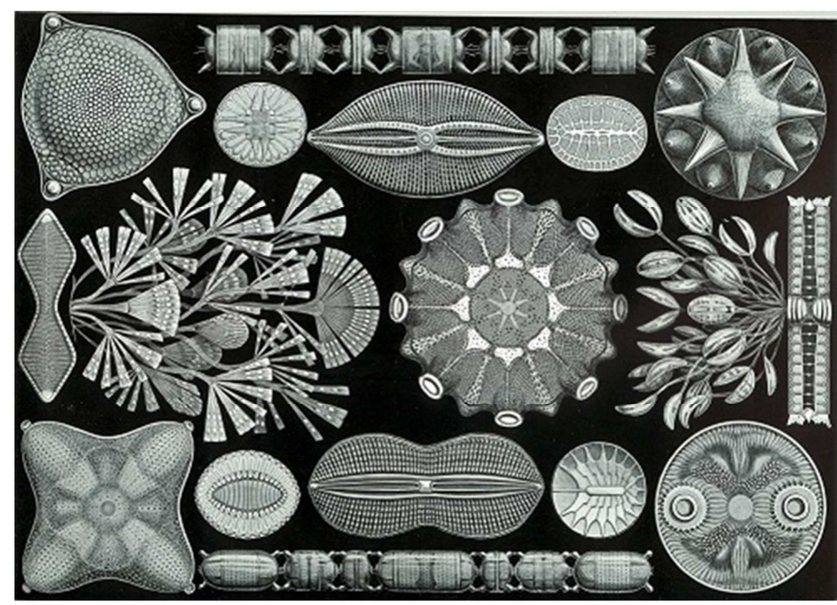

Figure 1. Different kinds of diatoms. The shells could be seen.

\section{Arguments to Propose as a Modelling Tool BPBH}

\subsection{Choice of a Mortality Rate}

The cell can divide or die. Here we mean death for disappearance from the population by means other than division. Diatoms are in the basis of the food chains. They have the ability of forming dispersed aggregates in the water column, which constitute the main food available to the early larval stages of many fish species. At such stages, larvae are passive and can only eat the prey passing in very close vicinity. This is the reason for assumption that the mortality $(1-\rho)$ is the same for all types.

\subsection{Choice the Particle's Lifespan Distribution}

From the birth to the splitting every cell goes through several specific stages known in advance and required a concrete time. Only after the successful completion of the cycle the cell is capable of dividing. Therefore we assume that the lifespan of the cell follows $N\left(\mu, \sigma^{2}\right)$.

\subsection{Arguments to Model Through BPBH}

(a) Only after completion of the whole cycle the cell is capable of dividing and every particle is of zero age at birth, that is to say $G(0)=G(0+)=0$. In this case of right continuity at the zero point of the d.f., the multidimensional g.f. is more suitable for research and analytical expression. The authors of all basic works unambiguously warn about that the g.f. of non Markov multidimensional BPBH can be impossible to express explicitly $[2,11]$.

(b) Every particle of the BPBH has a random life time $\tau$ and produces offspring just before dying. This coincides with the diatom's cell division mechanism.

(c) Impossibility for simultaneous birth and death of the particle together with producing offspring corresponds to the biological side.

(d) The mitosis results in one of the two daughters decreasing in size. $[1,12]$

(e) The diatoms stop dividing when their size drops below the minimum. $[1,12]$

(f) The size of the diatoms is between 30 and 150 microns. $[1,12]$

(g) The critical stop-dividing size is between half to onethird of the regular. $[1,12]$

(h) In discrete time the Fibonacci series describes successfully the diatoms' number. $[1,12]$

(i) The mortality $(1-\rho)$ is the same for all types.

(j) The indivisible cells have a bottom-down mechanism and may remain hidden for a long time.

The cell is a particle in the BHBP Z $(\mathrm{t})$ designated by $\mathrm{T}$.

\subsection{Data}

The authors consider data from samples taken from about 50 stations along the Bulgarian Black Sea coast during the summer of '94, '97, from 2002 to 2006, 2009 and 2011. The measured concentrations of chl-a reveal a distribution of a phytoplankton population localized to a particular depth and geographical longitude and latitude.

The authors offer an algorithm:

(Step 1.) Determine the endpoints of the rectangular parallelepiped contained the entire area.

(Step 2.) Set a desired step h.

(Step 3.) Divide the entire volume into $h^{3}$ equal subvolumes.

(Step 4.) Determine the endpoints of the all rectangular sub-parallelepipeds.

(Step 5.) Check each of the measured in the submitted data quantities in which sub-volume falls.

(Step 6.) Collect the registered quantities for each are

(Step 7.) Plot result.

(Step 8.) End.

The following diagram (Figure 2.) reflects the measured concentration of chl-a, giving rough guide about the distribution of the chl-a in the selected location. It gives an idea about the phytoplankton's distribution and from there of the diatoms, dominate kind of it on the Bulgarian Black See coast. 


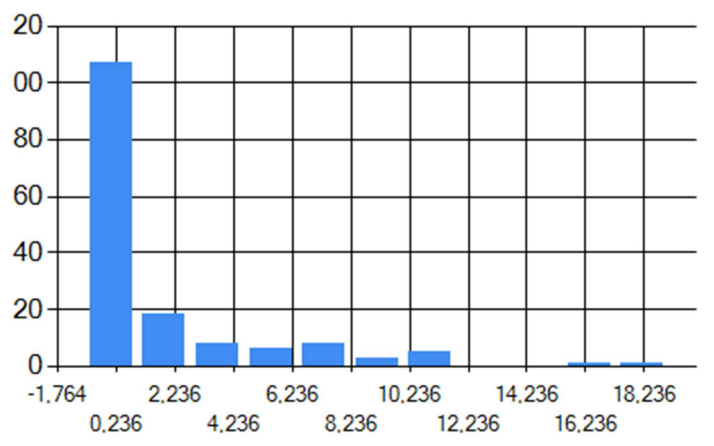

Figure 2. Measured concentration of chl-a.

\section{Diatoms Model}

Over the probability space $(\Omega, \mathfrak{J}, P)$ are defined a random variables

$\rho, \tau_{i}$ for $i=1, \ldots, 4$ and BPBH Z (t) with 4 types of particles, defined by theirs initial sizes. [6,8]. The particles' lifetimes are $\tau \in N\left(\mu, \sigma^{2}\right)$,

d.f. $G_{i}(t)=P(\tau \leq t)$. Taking into account $(\diamond 8)$ and the mechanism of the diatoms division, we get five as $\Delta$ subintervals number. $\Delta=[30,155]$ is divided as follows:

$$
\Delta^{\prime}{ }_{1}=[30,55], \Delta^{\prime \prime}{ }_{1}=(55,80], \Delta_{2}=(80,105], \Delta_{3}=(105,130],
$$

$\Delta_{4}=(130,150]$. Define: $\Delta_{1}=\Delta^{\prime}{ }_{1} \cup \Delta^{\prime}{ }_{1}$.

The BPBH with 4 types of the particles $T_{i} ; i=1, \ldots, 4$, depending on in which subintervals falls its initial sizes, is designated by

$$
Z(t)=\left(Z_{1}(t), Z_{2}(t), Z_{3}(t), Z_{4}(t)\right)
$$

Every particle is of zero age at the birth and it is impossible simultaneous birth, death and offspringproduction. This allows us the use of basic theorems for the multidimensional continuous non-Markov branching processes. It follows from what we said above that for the particles in the four-dimensional non-Markov BPBH Z ( $t$ ) are met:

Conditions for the particles in the four-dimensional nonMarkov BPBH Z (t):

Condition 1. The particle $T$ in $Z(t)$ represents diatom's cell. Its initial size defined the type.

Condition 2. The life span of $T$ is designated by $\tau \in$ $N\left(\mu, \sigma^{2}\right)$.

Condition 3. $T_{1}$ is in a not-splitting mode.

Condition 4. Accumulating sufficient critical mass, $T_{1}$ begins to merge with each other, generating a new $T_{4}$.

Condition 5. $T_{i} ; i=2,3,4$ reaches maturity with a

$$
\vec{r}=(\underbrace{r_{1}, \ldots, r_{n}}_{n}) ; \overrightarrow{0}=(\underbrace{0, \ldots, 0}_{\{n\}}) ; \overrightarrow{1}=(\underbrace{1, \ldots, 1}_{\{n\}}) ; \vec{s}=(\underbrace{s_{1}, \ldots, s_{n}}_{n})
$$$$
\vec{F}=(\underbrace{F_{1}, \ldots, F_{n}}_{n})
$$

$p_{i j}$ - the probability and respectively $m_{i j}$ the expectation $T_{j}$ to be a

$T_{i}$-descendant.

$m_{i j}(t)$ - the expected number $T_{j}$-descendants from one $T_{i}$ after an arbitrary time $t$ has passed.

Definition 4.1

$\mathrm{M}=\left\|\mathrm{m}_{\mathrm{ij}}\right\|_{i j}$ is the particle production mean matrix associated with the individual generating function (i.g.f.) $f$ (s), where:

$$
m_{i j}=\left.\frac{\partial f_{i}}{\partial s_{j}}\right|_{s=\overrightarrow{1}}
$$

probability $\rho$, when splits into $T_{i}$ and $T_{i-1}$ [9].

Condition 6. Each particle evolves independently of each other.

The mechanism of the particles division is illustrated by Figure 3.

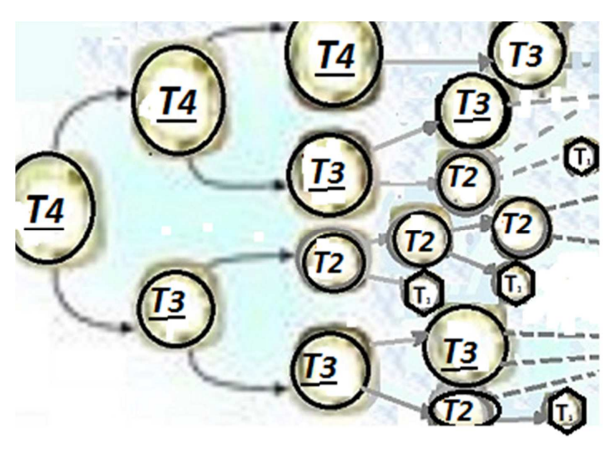

Figure 3. Mechanism of division of the particles.

\section{Equations of BPBH Z (t)}

Let us for any positive integer $n$ and $i, j=1, \ldots, n$ use the designations:

\section{Definition 4.2}

$\mathrm{U}(\mathrm{t})=\left\|\mathrm{m}_{\mathrm{ij}}(\mathrm{t})\right\|$ is the matrix of means at time $t \in[0, \infty)$.

Definition 4.3

$\|\mathrm{A}\|:=\max \left(\left|a_{i j}\right|\right)$ for any $n \times n$ matrix A.

Definition 4.4

The generating function (g. f.) $F(t, s)$ is

$$
\mathrm{F}(\mathrm{t}, \mathrm{s})=\sum_{r \in N_{n}} P\{Z(t)=r \mid Z(0)=\overrightarrow{1}\} s_{1}{ }^{r_{1}} \ldots s_{n}{ }^{r_{n}},
$$

where $N_{n}=\left\{\alpha=\left(\alpha_{1}, \ldots, \alpha_{n}\right): \alpha=0,1,2 \ldots\right\}$

Let us introduce the functions:

$$
\begin{gathered}
R_{i}\left(m_{i i}, u\right)=\sum_{n=1}^{\infty} m_{i i}{ }^{n-1}\left(G_{i}(t)\right)^{* n} \\
\left(G_{i}(t)\right)^{* 1}=G_{i}(\mathrm{t})
\end{gathered}
$$




$$
\left(G_{i}(t)\right)^{* n}=\int_{0}^{t} G_{i}^{*(n-1)}(t-u) \mathrm{d} G_{i}(u)
$$

Proposition 1.

For the particle production mean matrix $M$, associated with the individual generating function (i.g.f.) $f(s)$ we have:

$$
M=\left(\begin{array}{llll}
\rho & 0 & 0 & 0 \\
\rho & \rho & 0 & 0 \\
0 & \rho & \rho & 0 \\
0 & 0 & \rho & \rho
\end{array}\right)
$$

Proof:

From Condition $3-T_{1}$ is in a not-splitting mode and Condition 5 -

$T_{i} ; i=2,3,4$ reaches maturity with a probability $\rho$, when splits into $T_{i}$ and $T_{i-1}$

for the components of the i.g.f. we receive:

$$
\begin{gathered}
f_{1}(s)=1-\rho+\rho s_{1} \\
f_{i}(s)=1-\rho+\rho s_{i-1} s_{i} \text { for } i=2,3,4
\end{gathered}
$$

From definition 4.1 and from (1) follows the proposition.

\section{Expectation}

For the considered biological model $\|M\|<\infty$, therefore $\|U(t)\|$ is bounded on finite intervals, and $\|U(t)\|$ satisfies the matrix equation $[2,11]$ :

$$
U^{t}(t)=D\left[1-G^{*}(t)\right]+\int_{0}^{t} U^{t}(t-u) M^{t} \mathrm{~d}\left[G^{*}(u)\right]
$$

Where $D[x]$ is the diagonal matrix with $x_{i}$ in the i-th place, and $\mathrm{d}[G(u)]$ is the diagonal matrix with $d G_{i}(u)$ in the $i$-th entry. According $[2,11] U(t)$ is the unique solution of the equation (6), bounded on finite intervals.

$$
m_{i j}(t)=E\left\{Z_{j}(t) \mid Z(0)=e_{i}\right\}=\left.\frac{\partial F_{i}}{\partial s_{j}}\right|_{s=\overrightarrow{1}}
$$

Proposition 2.

For the moments of the non-Markov BPBH Z (t) with arbitrarily d.f. $G_{i}(t) i, j=1, \ldots, 4$ we have:

$$
m_{i j}(t)=1-G_{i}(t)+\rho \int_{0}^{t}\left(1-G_{i}(t-u)\right) d \sum_{n=1}^{\infty} \rho^{n-1}\left(G_{i}(t)\right)^{* n}
$$

$m_{i j}(t)$ for $\mathrm{i}<\mathrm{j}$ can be expressed from the his predecessor of $\mathrm{U}(\mathrm{t})$ sequentially on columns; $m_{i j}(t)=0$ for $i>j$.

Proof:

For the $F(t, s)$ it is valid $[2,11]$ :

$$
F(t, s)=\mathrm{s}\left(1-G_{i}(t)\right)+\int_{0}^{t} f(F(t-u, s)) d G(u)
$$

By components for $i=1, \ldots, 4$ we have:

$$
F_{i}(\mathrm{t}, \mathrm{s})=\mathrm{E}\left\{s^{Z(t)} \mid \mathrm{Z}(0)=e_{i}\right\}=\sum_{r \in N_{4}} P\left\{Z(t)=r \mid Z(0)=e_{i}\right\} s_{1}{ }^{r_{1}} \ldots s_{4}{ }^{r_{4}}
$$

From (5), (7) and (10), using the designation $F_{0}(t) \equiv 1$ we obtain:

$$
F_{i}(t, s)=s_{i}\left(1-G_{i}(t)\right)+(1-\rho) G_{i}(t)+\rho \int_{0}^{t}\left(F_{i}(t-u, s) F_{i-1}(t-u, s)\right) d G_{i}(u)
$$

From equations (7) and (11) for $i=2,3,4 j=1, \ldots, 4$ we receive:

$$
m_{i j}(t)=\delta_{i j}\left(1-G_{i}(t)\right)+\rho \int_{0}^{t} m_{i j}(t-u) F_{i-1}(t-u, s) d G_{i}(u)+\rho \int_{0}^{t} m_{(i-1) j}(t-u) F_{i}(t-u, s) d G_{i}(u)
$$

From definition 4.2:

$$
U^{t}(t)=\left(\begin{array}{clll}
m_{11}(t) & m_{21}(t) & m_{31}(t) & m_{41}(t) \\
0 & m_{22}(t) & m_{32}(t) & m_{42}(t) \\
0 & 0 & m_{33}(t) & m_{43}(t) \\
0 & 0 & 0 & m_{44}(t)
\end{array}\right)
$$

From equation (6):

$$
\begin{aligned}
& U^{t}(t)=\left(\begin{array}{ccccc}
1-G_{1}(t) & & 0 & 0 & 0 \\
0 & & 1-G_{2}(t) & 0 & 0 \\
& 0 & 0 & 1-G_{3}(t) & 0 \\
0 & 0 & 0 & 1-G_{4}(t)
\end{array}\right)+\int_{0}^{t} U^{t}(t-u) M^{t} \mathrm{~d}[G(u)]
\end{aligned}
$$

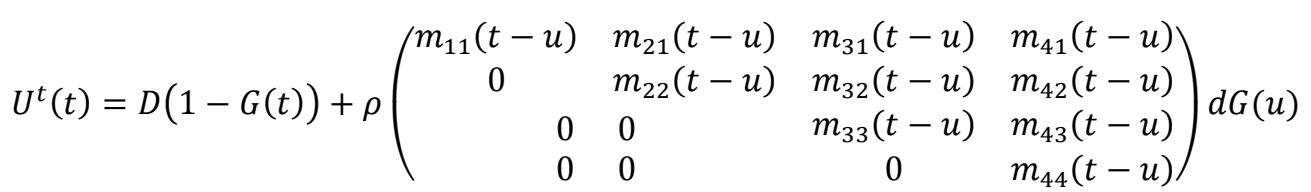




$$
+\rho\left(\begin{array}{ccccc}
0 & m_{11}(t-u) & m_{21}(t-u) & m_{31}(t-u) \\
0 & & 0 & m_{22}(t-u) & m_{32}(t-u) \\
& 0 & 0 & 0 & m_{33}(t-u) \\
& 0 & 0 & 0 & 0
\end{array}\right) d G(u)
$$

Therefore for $i=1, \ldots, 4$ we have:

$$
m_{i i}(t)=1-G_{i}(t)+\rho \int_{0}^{t} m_{i i}(t-u) d G_{i}(u)
$$

For $j=2,3,4$ :

$$
\begin{aligned}
& m_{j 1}(t)=\rho \int_{0}^{t} m_{(j-1) 1}(t-u)+m_{j 1}(t-u) d G_{i}(u) \\
& \text { For } j=3,4: \\
& m_{j 2}(t)=\rho \int_{0}^{t} m_{(j-1) 2}(t-u)+m_{j 2}(t-u) d G_{i}(u) \\
& m_{43}(t)=\rho \int_{0}^{t} m_{33}(t-u)+m_{43}(t-u) d G_{i}(u)
\end{aligned}
$$

For $i=1, \ldots, 4$ with $0<j<i$ :

$$
m_{i j}(t)=0
$$

We solve equations as follows:

(14) are renewal equations. Using designation (2) for their solutions we have the formula, presented in $(19)[2,11]$ :

$$
m_{i i}(t)=1-G_{i}(t)+m_{i i} \int_{0}^{t}\left(1-G_{i}(t-u)\right) d R_{i}\left(m_{i i}, u\right)
$$

From equations (14) we get $m_{11}(t)$ and substitute it in equations (15) with $\mathrm{j}=2$, obtaining renewal equation in general form. Then replace the obtained for $m_{21}(t)$ in equations (15) for $\mathrm{j}=3$, and so on. That way we can get all the expectations. From the recursion above follows the proposition [10].

\section{Discussion}

Mathematically more convenient is the choice of discrete process or continuous process with $\tau \in \operatorname{Exp}(\lambda)$ because the process would be Markov. The multidimensional continuous non-Markov process with d.f. $\tau \in N\left(\mu, \sigma^{2}\right)$, fits better on the biological side. We could assume, that bisection of the cells occurs approximately when they double, it is logical and rom a biologic point of view

The derived results for the expectations would give a better idea about the expected population size after any time has elapsed.

From a biologic point of view, to seek the asymptotic behavior of the number of diatoms after an infinite period of time is of no interest, of course. For this reason, the authors decided to miss it. The mathematical task for the asymptotic behavior of the particle number could be derived for $Z(t)$. Some results for the asymptotic of BPBH with normally distributed life span of the particles are obtained in [13, 14].

Supplementing the branching properties by controlling the number of progenitors in every generation allows for modelling a random migratory movements in and out of the population [15].

\section{Conclusions}

The most characteristic feature of the diatoms is their cell wall. This shell is the reason of mitosis to result in one of the two daughters decreasing in size. This is the reason the authors to determine the particle's type by its initial size.

The phytoplankton consists of microscopic aquatic species, both fresh water and marine. Mostly predominate diatoms and others. The purpose of this work is to model the dynamics of a diatoms population, one of the most ancient inhabitants of our planet. The formulated mathematical model would give a better idea about the expected population size in the near and further future. The phytoplankton and in particular a diatoms play a key role at the base of the food chains, climate regulation and ecology.

When the condition one of the two daughters decreasing in size met is, the model could be applicable for populations of unicellular organisms.

Only after completion of the whole cycle the cell is capable of dividing, which corresponds to the mathematical condition of right continuity in the zero point of the distribution function. This respectively makes the g.f. more suitable for research and analytical expression. In general, g.f. are very difficult to express in an explicit form, which the authors of all the basic works are unambiguously warned about $[2,11]$. Every particle of the BPBH has a random life time, producing offspring, just before dying. These two conditions completely coincide with the cell-division and this is one of the reasons why the authors are turning to model through $\mathrm{BPBH}$.

The phytoplankton cells have the ability of forming dispersed aggregates in the water column, which constitute the main food available to the early larval stages of many fish species. At such stages, larvae are passive and can only eat the prey passing in very close vicinity. This is the reason for our assumption that the mortality $(1-\rho)$ is the same for all types.

As a modelling tool we propose the BPBH.

Impossibility for simultaneous birth and death of the particle together with producing offspring corresponds to the biological side.

The moments are expressed. The matrix equation, corresponding to the system of sixteen integral equations was determined.

\section{Acknowledgements}

The authors are grateful to Prof. Snejana Moncheva for hers collaboration for the elaboration of the database and hers valuable remarks in the field of marine biology.

The authors are thankful to the anonymous referee, kindly 
revised the manuscript, for the precise and useful guidance on well paper organization.

\section{References}

[1] Bowler Ch., De Martino A., Falciatore A. (2010) Diatom cell division in an environmental context. Elsevier/Plant Biology. Vol. 13, pp. 623-630.

[2] Harris T. E. (2002) The Theory of Branching Processes. Dover Publications.

[3] Denaro G., Valenti D., La Cognata A., Spagnolo B., Bonanno A., Basilone G., Mazzola S., Zgozi S. W., Aronica S., Brunet C. (2013) Spatio-temporal behavior of the deep chlorophyll maximum in Mediterranean Sea: Development of a stochastic model for phytoplankton dynamics. Ecological Complexity, 13 21-34.

[4] Silvestrov S., Malyarenko A., Rancic M. (2018) Stochastic Processes and Applications. Springer Proceedings in Mathematics \& Statistics.

[5] Kasprzak P., Padisak J., Koschel R., Krienitz L., Gervais F. (2008) Chlorophyll a concentration across a trophic gradient of lakes: An estimator of phytoplankton biomass? Elsevier/Management of Inland Waters. Vol. 38, pp. 327-338.

[6] Terzieva A. June (2016) Model phytoplankton population by branching processes, In: Biomath Communications, Vol. 3, Issue 2, ISSN 2367-5233 (Print) 2367-5241 (Online).

[7] Haccou P., Jagers P., and Vatutin V. A. (2005) Branching Processes: Variation, Growth, and Extinction of Populations. Cambridge University Press.
[8] Terzieva A. (2013) Modeling of the amount of chlorophyll-a, contained in the phytoplankton population by branching process, MIE 2013: Doctoral Conference in Mathematics, Informatics and Education, (2013) Sofia, Bulgaria.

[9] B. A. Sevastyanov (2016) A class of subcritical branching processes with immigration and infinite number of types of particles. In: Discrete mathematic and applications. Vol. 17. Issue 1. Schmidt Periodicals GmbH, Bad Feilnbach https://doi.org/10.1515/DMA.2007.001.

[10] A. Terzieva (2015) Model population dynamics by branching stochastic processes with three types of particles, International conference: Mathematics as a fundamental and applied science, Varna, Bulgaria.

[11] Athreya K. B., Ney P. E. (2004) Branching processes. Dover Publications Inc., ISBN 10: 0486434745 - ISBN 13: 9780486434742 .

[12] Raven P., Evert R., Eichhorn S. (2006) Biologie der Pflancen/15.6.2 Diatomeen (Kieselalgen). Walter de Gruyter, Berlin. New York. (In German).

[13] Terzieva A. (2015) Model of phytoplankton by branching processes, 4-th Advanced Research in Scientific Areas ARSA, Zilina, Slovakia.

[14] Terzieva A., Terziev G. (2015) Multitype branching process as model for phytoplankton population and chlorophyll-a contained therein, In: American Review of Mathematics and Statistics, Vol. 3, Issue 1, ISSN 2374-2348 (Print) 2374-2356 (Online).

[15] Miguel González Velasco, Ines Maria Garcia del Puerto, George Yanev Petrov (2017) Controlled branching processes. Elsevier. Vol. 2. 